\title{
An immunomagnetic cell separation system based on a retroviral vector containing a chimeric, recombinant human-murine CD4 gene
}

\author{
PATRYK GÓRNIAK, PRZEMYSŁAW JUSZCZYŃSKI
}

Institute of Hematology and Transfusion Medicine, Warsaw, Poland

\begin{abstract}
Primary B-and T-lymphocytes and neoplastic cells of B-and T-cell origin are particularly hard to transfect with plasmids. Thus, functional and molecular studies in immunology and oncohematology require selection of cells after plasmid transduction, assuring obtaining homogeneous cell populations expressing plasmid-delivered molecules. The selection is usually achieved by incorporation of an antibiotic resistance gene or fluorescent protein into a plasmid and subsequent antibiotic selection or fluorescence-activated cell sorting (FACS). However, these cell sorting methods have significant drawbacks that limit their utilization. An ideal system should be fast, cheap and ensure efficient selection of transfected cells. In an attempt to deliver a sorting system meeting these requirements, we have generated a plasmid for retroviral gene delivery and expression, containing a recombinant human-murine CD4 gene, with a truncated intracellular domain to prevent undesired signaling events. After retroviral infection, cells expressing hmCD4 are selected by immunomagnetic anti-mCD4 beads. The system offers a very robust, efficient, fast and cost-saving cell separation solution. The vector is available for the academic community upon request.
\end{abstract} beads.

Key words: cell selection, immunomagnetic selection, cell transfection, CD4, immunomagnetic

(Centr Eur J Immunol 2018; 43 (3): 353-357)

\section{Introduction}

Selection of transfected cells is usually a necessary procedure to obtain homogeneous cell populations suitable for studying functions of plasmid-delivered molecules [1]. The efficient selection procedure is particularly essential for experimental/functional studies in hard-totransfect cells, such as lymphoma cells or primary B- or T-lymphocytes. Depending on the method and cell type, real-life transfection efficiencies in laboratory practice typically do not exceed $10-40 \%$. One of the most widely used procedures for selection of cells after plasmid transfection is incubation with cytotoxic antibiotics that eliminate untransfected cells, while sparing cells carrying a vector containing an antibiotic resistance gene. However, this method has considerable disadvantages. First and foremost, it is not acceptable for primary cells (including primary lymphoma cells) due to spontaneous apoptosis of these cells in vitro. Second, if the delivered gene's product is toxic or has a negative effect on cell growth, selection of cells expressing that gene cannot be achieved [1]. Third, metabolites of antibiotics can be biologically active and alter the behavior of studied cells [2, 3]. Last but not least, isolation of a homogeneous population of cells expressing a gene by antibiotic selection is very time consuming; thus the selection procedure allows the cells to develop mechanisms compensating the biological effects of the overexpressed molecule [4]. Transfection with vectors that instead of an antibiotic-resistance gene carry fluorescent proteins such as green fluorescent protein (GFP) can be a solution to some of these limitations [5, 6]. Cells transfected with such a vector are subsequently sorted with a high-speed fluorescence-activated cell sorter (FACS). Although this methodology is generally widely accessible, prolonged sorting periods can be toxic and increase the risk of bacterial or fungal contamination. Each of the commonly used systems has drawbacks that limit its utilization. An ideal system should be fast, cheap and ensure efficient selection of transfected cells.

Correspondence: Prof. Przemysław Juszczyński, Institute of Hematology and Transfusion Medicine, 14 I. Gandhi St., 02-776 Warsaw, Poland, e-mail: pjuszczynski@ihit.waw.pl

Submitted: 21.06.2018; Accepted: 16.08.2018 
In an attempt to deliver a sorting system meeting these requirements, we have generated a retroviral plasmid for gene expression in human cells, containing a recombinant, chimeric, human-murine $\mathrm{CD} 4$ gene which enables expression of this protein on plasma membrane of transfected cells. After transduction, cells are sorted using immunomagnetic selection with anti-mouse CD4 antibody conjugated with magnetic beads.

\section{Material and methods}

\section{Cell lines, cell culture and chemicals}

Human lymphoma cell lines Karpas 422 and DHL4 were purchased from ATCC and maintained in RPMI-1640 (Lonza, Basel, Switzerland). The HEK-293T cell line was purchased from ATCC and maintained in DMEM (Lonza, Basel, Switzerland), supplemented with $10 \mathrm{mM}$ HEPES buffer, $50 \mathrm{U} / \mathrm{ml}$ penicillin, $50 \mathrm{U} / \mathrm{ml}$ streptomycin and $10 \%$ heat-inactivated FBS (Lonza, Basel, Switzerland). Cells were grown in a humidified atmosphere at $37 \mathrm{~s} \mathrm{C}$ with $5 \% \mathrm{CO}_{2}$.

\section{Generation of recombinant human-murine CD4 gene and its subcloning}

cDNA encoding recombinant chimeric human-mouse CD4 (hmCD4) was generated in a multistep PCR process. At first, the murine CD4 fragment containing complete transmembrane and extracellular regions was amplified (For1: 5'ACTCAAGGGAAGACGCTGGTGC3'; Rev1: 5'CTGTTGGTGCCGGCACCTGA3') using pBS mCD4 plasmid (Addgene, Cambridge, MA, USA) as a template. Next, the human CD4 leader fragment was added in the second PCR reaction, performed with forward primer containing the complete human leader sequence (For2: 5'ATGAACCGGGAGTCCCTTTTAGGCACTTGCTTCTGGTGCTGCAACTGGCGCTCCTCCCAGCAGCCACTCAAGGGAAGACGCTGGTGC3'; Rev2: 5'CTGTTGGTGCCGGCACCTGACACAGCAGAG3'). AgeI and DraIII restriction sites and stop codon were added in the last PCR reaction (For3: 5' ACTGACCGGTATGAACCGGGGAGTCCCTTTAGGC3'; Rev3: 5'ACTGCACCAGG TGCTACTGTTGGTGCCGGCACCTG3'). The gel-purified hmCD4 fragment and pLPCX (Addgene, Cambridge, MA, USA) were digested using AgeI and DraIII (New England Biolabs, Ipswich, MA, USA) restriction enzymes and ligated to yield the pLPCX-hmCD4 construct (termed pLPCD4). pLPCD4-GFP was constructed as follows: GFP (Green Fluorescence Protein) cDNA with restriction sites for EcoRI and ClaI was generated by PCR reaction (For4: 5'AGTCATCGATTTACTTGTACAGCTC3'; Rev4: 5'AGTCGAATTCATGGTGAGCAAGGGC3') with the pMIG vector (Addgene, Cambridge, MA, USA) used as a template. The GFP fragment was cloned into MCS of the pLPCD4 construct at EcoRI and ClaI sites (New England Biolabs, Ipswich, MA, USA) to yield pLPCD4-GFP. PCR reactions were performed using Color Pfu Plus DNA Polymerase (Eurx, Gdansk, Poland). All sequences were confirmed to be correct with direct, bidirectional Sanger sequencing.

\section{Retroviral infection}

Generation of retroviruses and infection was performed as described previously [7]. Briefly, HEK-293T cells were transfected with pKAT, VSV-g and a retroviral plasmid encoding the hmCD4 transgene using Lipofectamine 2000 reagent (Invitrogen, Carlsbad, CA, USA). Following $24 \mathrm{~h}$ incubation at $37^{\circ} \mathrm{C}$, the retroviral supernatant was collected, mixed with hexadimethrine bromide $(8 \mu \mathrm{M}$ final concentration; Sigma-Aldrich, St. Louis, MO) and used to infect HEK-293T, Karpas 422 or DHL4 cells.

\section{Immunomagnetic cell separation}

Positive immunomagnetic cell separation was performed using a Miltenyi Biotec mouse CD4 kit according to the manufacturer's instructions. Briefly, cells were collected and resuspended in $90 \mu \mathrm{l}$ of degassed, $\mathrm{Ca}^{2+}$ - and $\mathrm{Mg}^{+2}$-free PBS supplemented with $0.5 \%$ BSA (MACS buffer). Cells were next labeled with anti-CD4 (L3T4) magnetic MicroBeads at a $1 / 10$ dilution for $15 \mathrm{~min}$ at $4{ }^{\circ} \mathrm{C}$. Cells were washed, centrifuged at $300 \times \mathrm{g}$ for $10 \mathrm{~min}$, and resuspended in $500 \mu \mathrm{l}$ of MACS buffer. The cell suspension was then applied to a MACS $\mathrm{MS}^{+}$separation column placed in the magnetic field of a MACS Separator (Miltenyi Biotec, Auburn, CA, USA), washed three times with MACS buffer. After washing out unlabeled cells, the column was removed from the magnetic field and the magnetically retained hmCD4+ cells were eluted in $1 \mathrm{ml}$ of MACS buffer as the positively selected cell fraction.

\section{Flow cytometry}

Evaluation of GFP and chimeric human-mouse (hm) CD4 expression was performed using a FACS Canto flow cytometer. For hmCD4 staining, anti-mouse monoclonal PE-conjugated CD4 antibody (GK1.5, Biolegend, San Diego, CA) was used.

\section{Bioinformatic analysis}

Analyses confirming the location of signal peptide cleavage sites in an amino acid sequence was performed by means of SignalP 4.1 Server (http://www.cbs.dtu.dk/services/SignalP/). The method incorporates a prediction of cleavage sites and a signal peptide/non-signal peptide prediction based on a combination of several artificial neural networks [8].

\section{Results}

We first generated a recombinant chimeric humanmouse CD4 ( $h m C D 4)$ gene containing murine transmem- 

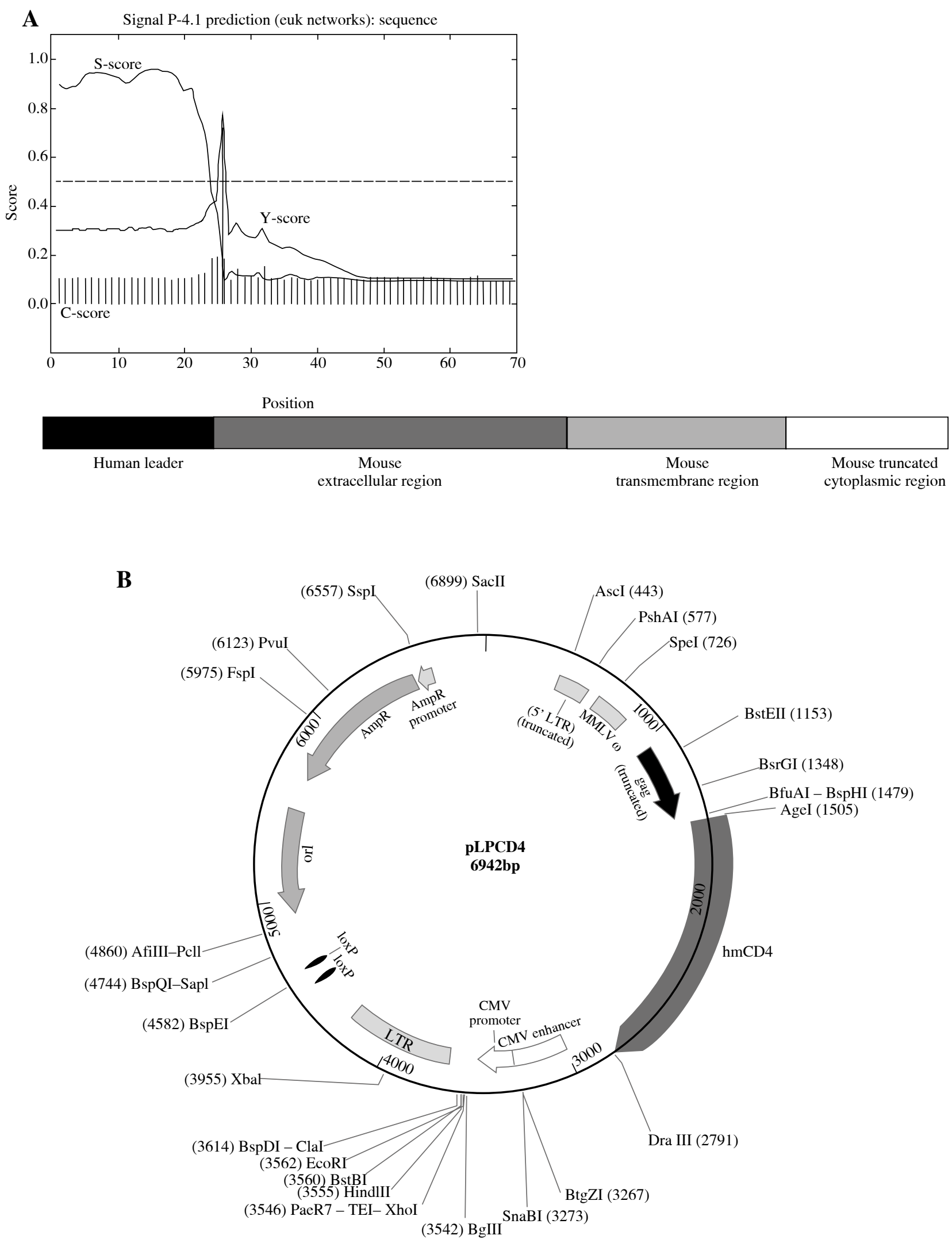

Fig. 1. Generation of pLPCD4 vector. A) Schematic diagram of a chimeric hmCD4 gene. Presence of functional signal peptide and location of signal peptide cleavage sites in hmCD4 were confirmed using SignalP 4.1 software. B) Map of pLPCD4 construct 
A

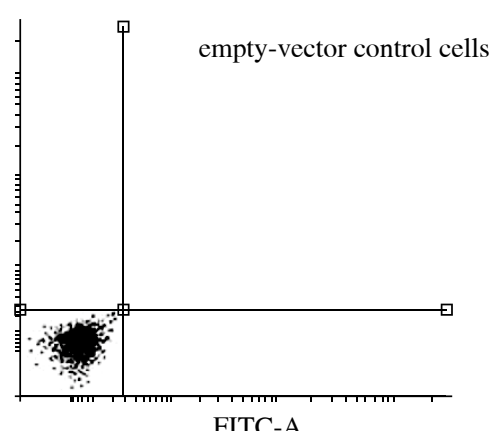

FITC-A

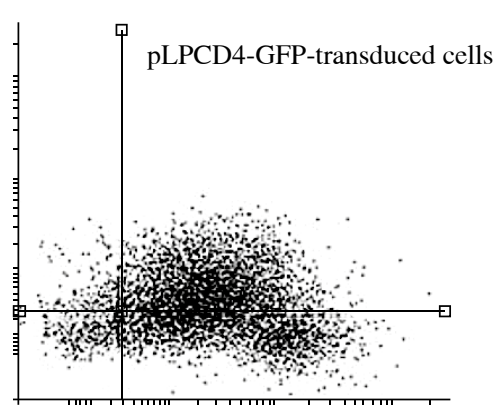

FITC-A
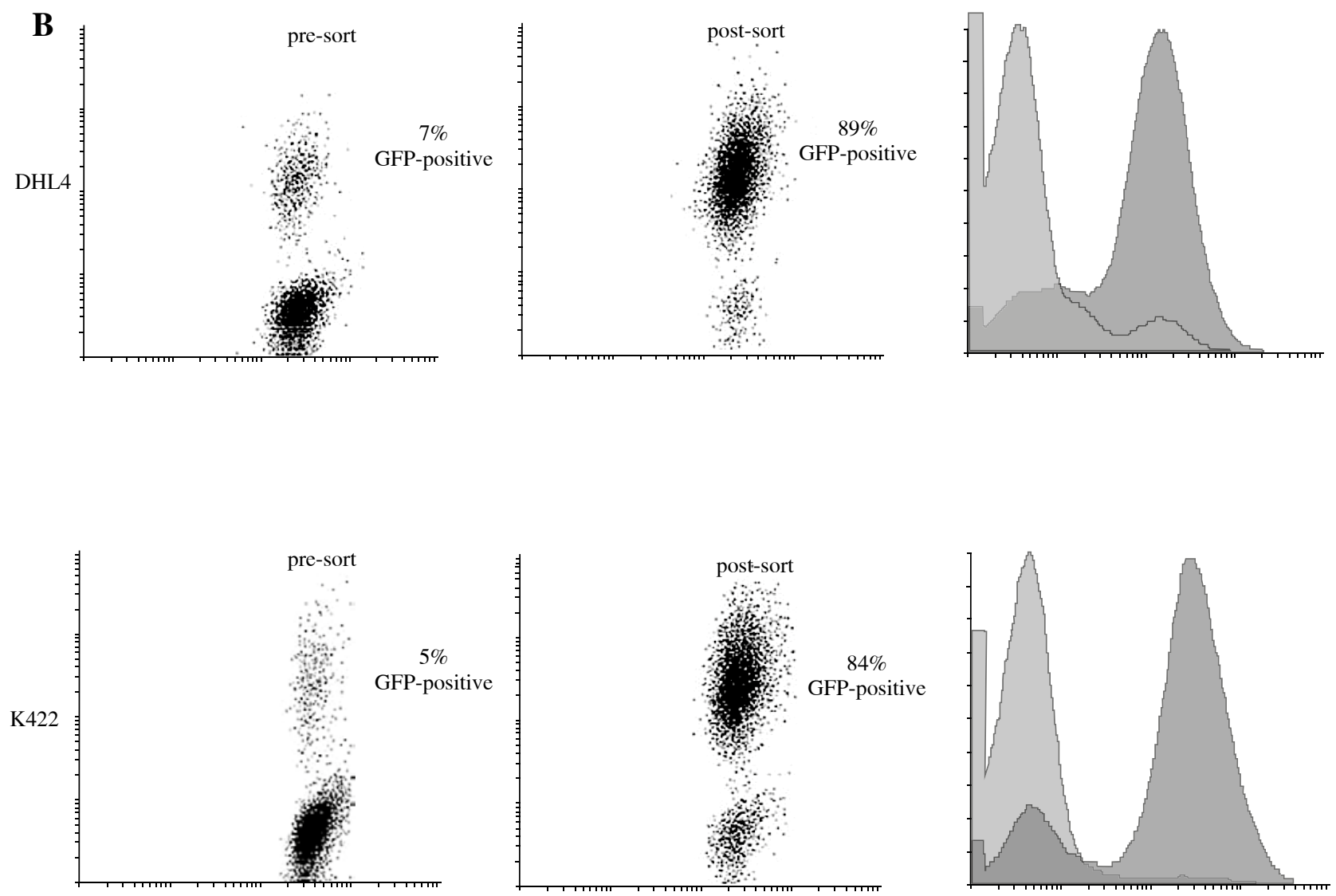

\footnotetext{
$\square$ pre-sort

post-sort
}

Fig. 2. A) Co-expression of hmCD4 protein and GFP protein in HEK-293T cells. Cells were infected with either emptyvector virus or pLPCD4-GFP. CD4 surface expression (PE-labeled antibody) and GFP fluorescence were assessed by flow cytometry. B) Analysis of selection efficiency in K422 and DHL4 cells, retrovirally transduced with pLPCD4-GFP vector and immunomagnetically sorted using CD4 (L3T4) MicroBeads. Fraction of GFP-positive cells pre- and post-sort was evaluated by flow cytometry 
brane and extracellular regions. The use of a murine CD4 portion allows for specific selection of cells transfected with a vector, regardless of the presence of human CD4. The cytoplasmic fragment of murine origin was truncated to prevent association with endogenous SRC family kinases and CD4-associated signaling in transduced cells [9]. The recombinant gene contains a human leader peptide, responsible for trafficking of CD4 to the plasma membrane. Presence of a functional signal peptide and location of the signal peptide cleavage site in hmCD4 was confirmed by bioinformatic analysis with SignalP 4.1 software (Fig. 1A). Thereafter, the chimeric $h m C D 4$ gene was used to replace the puromycin resistance gene in a retroviral vector of Moloney Murine Leukemia Virus (MoMuLV) origin. The $h m C D 4$ was placed under the control of the 5'LTR promoter. A map of this construct (termed pLPCD4) is depicted in Figure 1B. To assess the vector performance, we cloned the GFP reporter gene into multiple cloning site (MCS) of pLPCD4 (pLPCD4-GFP). The pLPCD4-GFP vector was then used to generate retroviral particles and infect HEK-293T cells. The cells exhibited robust membrane expression of hmCD4 and coexpression of GFP, confirming proper construction of $\mathrm{hmCD} 4$ and vector assembly (Fig. 2A). Next, lymphoma cell lines Karpas 422 and DHL4 were retrovirally transduced with the pLPCD4-GFP. As expected, infection resulted in less than $10 \%$ of GFP+ cells (Fig. 2B). Cells were then sorted with the CD4 (L3T4) MicroBeads Kit and efficacy of the selection method was evaluated by flow cytometry; results are shown in Figure 2B. In the case of the DHL4 cell line, our system allowed for about a 13-fold increase in GFP-positive cells (7\% and $89 \%$ of GFP-positive cells before and after selection, respectively), and about a 17-fold increase in GFP-positive cells in the case of the Karpas 422 cell line $(5 \%$ and $84 \%$ of GFP-positive cells before and after selection, respectively).

\section{Discussion}

We describe herein the construction of a pLPCD4 retroviral plasmid for gene expression and immunomagnetic selection in human hard-to-transfect cells. The pLPCD4 plasmid contains a recombinant, chimeric, human-murine CD4 gene which enables expression of this protein on the plasma membrane of transfected cells and subsequent cell sorting using immunomagnetic selection with anti-mouse CD4 antibody conjugated with magnetic beads. The developed selection system is efficient, very fast and cost-saving. The manual cell selection procedure takes up to 1 hour and does not require additional equipment. After the quick immunomagnetic sorting, the population of plasmid-transduced cells was enriched markedly, yielding $84-89 \%$ of GFP-positive cells. Of note, this level of enrichment is acceptable for most biological assays. In addition, the speed of the procedure ensures that unlike with FACS or antibiotic selection, hmCD4 sorted cells are exposed to potentially harmful/toxic conditions for a very limited time, which might significantly influence the cell condition after the selection. Accordingly, lymphoma cells after immunomagnetic sorting typically exhibited viability $\geq 80-85 \%$. Importantly, since the recombinant hmCD4 lacks a functional intracellular domain, its expression does not trigger cellular signaling and does not interfere with the function of the studied plasmid-delivered molecule.

\section{Conclusions}

All considered, the method described herein can be very helpful, particularly when working with hard-totransfect cells, such as lymphoma cells. Nonetheless, the immunomagnetic selection method using the pLPCD4 vector is certainly not limited to addressing the difficulties with transfection efficiency; it can be a method of choice, replacing FACS in experiments with cells exhibiting intrinsic fluorescence or treated with fluorescent compounds. Compared to FACS and antibiotic selection, this approach represents a simple, time-saving and cost-efficient method. The vector is available for academic users upon request.

The authors declare no conflict of interest.

\section{References}

1. Mortensen RM, Kingston RE (2009): Selection of transfected mammalian cells. Curr Protoc Mol Biol Chapter 9: Unit 9.5.

2. Oliva-Trastoy M, Defais M, Larminat F (2005): Resistance to the antibiotic Zeocin by stable expression of the Sh ble gene does not fully suppress Zeocin-induced DNA cleavage in human cells. Mutagenesis 20: 111-114.

3. Valera A, Perales JC, Hatzoglou M, Bosch F (1994): Expression of the neomycin-resistance (neo) gene induces alterations in gene expression and metabolism. Hum Gene Ther 5: 449456.

4. Feng L, Vijayasankaran N, Shen A, et al. (2010): Cell culture processes for monoclonal antibody production. MAbs 2 : 466-479.

5. Chalfie M, Tu Y, Euskirchen G, et al. (1994): Green fluorescent protein as a marker for gene expression. Science 263: 802-805.

6. Misteli T, Spector DL (1997): Applications of the green fluorescent protein in cell biology and biotechnology. Nat Biotechnol 15: 961-964.

7. Szydlowski M, Kiliszek P, Sewastianik T, et al. (2016): FOXO1 activation is an effector of SYK and AKT inhibition in tonic BCR signal-dependent diffuse large B-cell lymphomas. Blood 127: 739-748.

8. Petersen TN, Brunak S, von Heijne G, Nielsen H (2011): SignalP 4.0: discriminating signal peptides from transmembrane regions. Nat Methods 8: 785-786.

9. Palacios EH, Weiss A (2004): Function of the Src-family kinases, Lck and Fyn, in T-cell development and activation. Oncogene 23: 7990-8000. 